\title{
The Void in Clinician Counseling of Cannabis Use
}

\author{
Susan L. Calcaterra, MD, MPH, MS ${ }^{\top}$, Chinazo O. Cunningham, MD, $M S^{2}$, and \\ Christian J. Hopfer, $M D^{3}$
}

'Department of Medicine, Division of General Internal Medicine, University of Colorado School of Medicine, Anschutz Medical Campus, Aurora, CO, USA; ${ }^{2}$ Division of General Internal Medicine, Montefiore Medical Center/Albert Einstein College of Medicine, The Bronx, NY, USA; ${ }^{3}$ Department of Psychiatry, University of Colorado Anschutz Medical Campus, Aurora, CO, USA.

As more states legalize cannabis for medical use, people increasingly use cannabis to treat medical conditions. Well-documented harms of cannabis use include increased risk of fatal auto accidents, neurocognitive deficits, and increased risk of addiction. Observational data supports the use of cannabis for pain, nausea and vomiting related to chemotherapy, and multiple sclerosis spasticity symptoms. Given potential harms versus benefits of cannabis use, how should physicians counsel patients regarding their cannabis use? This paper briefly reviews the evidence supporting medical cannabis use for pain. We consider cannabis use as a harm reduction strategy for pain management. We encourage routine, longitudinal assessments of cannabis use among patients. We discuss the commercialization of cannabis for financial gain, contributing to potent and addictive cannabis. We highlight the concerning phenomena of cannabis dispensary workers as proxy clinicians. Finally, we present three strategies to reduce public harms associated with potent cannabis use including required testing and reporting of tetrahydrocannabinol/cannabidiol concentrations, rigorous study of high-potency cannabis available for purchase in dispensaries across the USA, and large-scale efforts to measure cannabis consumption in medical records so prospective, longitudinal studies can be conducted to correlate consumption measures with medical and psychiatric outcomes.

J Gen Intern Med 35(6):1875-8

DOI: $10.1007 / \mathrm{s} 11606-019-05612-4$

(๑) Society of General Internal Medicine 2019

$\mathrm{P}$ rimary care physicians play an important role in screening, identifying, and treating patients with unhealthy substance use. The United States Preventive Services Task Force (USPSTF) recommends that primary care clinicians perform screening and brief intervention for unhealthy alcohol and tobacco use. ${ }^{1,2}$ Increasingly, patients use cannabis to treat a myriad of medical conditions despite limited scientific evidence about the efficacy of cannabis use. ${ }^{3}$ How should

Received September 3, 2019

Accepted December 6, 2019

Published online January 2, 2020 clinicians counsel patients on the risks or benefits of cannabis use? Known harms of cannabis use include increased risk of fatal auto accidents, ${ }^{4}$ neurocognitive deficits, ${ }^{5}$ and increased risk of addiction. ${ }^{6}$ In addition, cannabis use has changed substantially over the past decades, with new formulations and high-potency products. It is unknown whether data from studies examining lower potency products generalize to higher potency formulations. Further complicating the issues is the widespread marketing of cannabis to treat various medical and mental health conditions in states with legal medical cannabis and on online dispensary websites. ${ }^{7}$ With legalization of cannabis for medical and recreational use, the number of people using cannabis-containing products is likely to increase. As with any other addictive substance, it is important that clinicians provide their patients with evidence-based recommendations to prevent or reduce adverse health effects related to cannabis use. To date, such recommendations are not available.

\section{EVIDENCE SUPPORTING MEDICAL CANNABIS USE}

Despite the known harms associated with cannabis use, there is evidence for the medical benefit of cannabis. The Food and Drug Administration (FDA) has approved two synthetic cannabinoids for the treatment of chemotherapy-associated nausea and vomiting (dronabinol and nabilone) and AIDS-related wasting syndrome (dronabinol). In 2018, the FDA approved cannabidiol (CBD), a cannabinoid derived from the cannabis plant, for the treatment of severe childhood-onset epilepsy. FDA approval followed research demonstrating that the use of CBD was safe and efficacious to reduce convulsant seizures among children and young adults with severe, intractable, childhood-onset, treatment-resistant epilepsy. ${ }^{8,} 9$ Several studies have examined cannabis use to treat medical conditions, including pain, spasticity, and chemotherapy-induced nausea and vomiting. In 2017, the National Academies of Sciences, Engineering, and Medicine reviewed the current state of evidence for medical cannabis and concluded, "there is conclusive or substantial evidence that cannabis or cannabinoids are effective for the treatment of pain, chemotherapy-induced nausea and vomiting, and for improving patient-reported multiple sclerosis spasticity symptoms." 10 Many of the studies examined demonstrated a trend for benefit, but lacked 
statistical significance. This may be due to the variety of cannabinoid products used (flower versus synthetic cannabinoids), to the inclusion of subjective versus objective outcome measures (self-report versus scale measurements for spasticity), or due to combining studies examining various conditions (neuropathic versus chronic versus cancer pain). Despite the methodological shortcomings noted in these studies, there is evidence to suggest that cannabis has a role in the treatment of pain and thus as an opioid-sparing agent. ${ }^{11}$

\section{CANNABIS FOR PAIN MANAGEMENT}

Many patients already use cannabis for pain management. Understanding when cannabis use may be indicated for pain can inform clinician's practice recommendations. Cannabis could be considered in patients with pain who have failed or have contraindications for traditional non-opioid pain medications, in combination with physical therapy, thermotherapy, acupuncture, ${ }^{12}$ and other non-pharmacotherapies. While no studies have conducted head-to-head trials of opioids versus cannabis for pain, medical cannabis may be preferred over opioid pain relievers for patients who experience minimal or no benefits from escalating doses of opioids or for patients with opioid-related adverse events. Patients at increased risk of opioid overdose, including those with a prior overdose or a substance use disorder, use of high opioid dosages $(\geq 90$ MME/day), or concurrent benzodiazepine use, may have the greatest reduction in risk with a transition from opioid pain reliever to medical cannabis for pain management or as a method to reduce their total daily opioid use. Clinicians should also inform their patients that, given the federal restrictions on cannabis-related research, little is known about the long-term benefits or harms with cannabis use for pain management.

\section{MEDICAL CANNABIS, OPIOID PAIN RELIEVERS, AND OPIOID OVERDOSE DEATHS}

Much has been published about the relationship between cannabis use, opioid pain medications, and opioid overdose deaths. Initial studies demonstrated that US states with medical cannabis laws had reductions in prescribed opioid daily doses as compared with states without medical cannabis laws. ${ }^{13,}{ }^{14}$ Similarly, states with medical cannabis laws reported fewer opioid overdose deaths than would be expected. ${ }^{15,16}$ A subsequent study replicated these findings between 1999 and 2013, but following 2013, overdose deaths in states with medical cannabis laws reversed direction and remained positive. ${ }^{17}$ The reversal in opioid overdose deaths in states with medical cannabis laws may be due to a shift in the opioid epidemic, initially fueled by opioid pain relievers, then transitioning to overdose deaths more frequently attributed to heroin and fentanyl. ${ }^{18}$ Given an ecological association between cannabis use and a reduction in opioid pain reliever use, patients may consider cannabis use for pain management as a harm reduction technique to reduce their total daily opioid use.
Patients should be counseled about risks related to regular daily cannabis use, such as the development of cannabis withdrawal syndrome when cannabis use is abruptly stopped. Commonly reported symptoms of cannabis withdrawal include anxiety or nervousness, hostility, insomnia, depressed mood, restlessness, and headache. ${ }^{19}$ Finally, regular cannabis use may lead to the development of problematic use, known as cannabis use disorder. Data from the National Survey of Drug Use and Health (NSDUH) revealed that $27.2 \%$ of the US population reported daily cannabis use, and of these, approximately $10 \%$ had some degree of cannabis use disorder. ${ }^{20}$ Taking these data into account, physicians should encourage patients to weigh risks and benefits when considering daily cannabis use for pain management. If there is concern that patients on chronic opioid therapy have developed an opioid use disorder, medications like methadone or buprenorphine effectively treat opioid use disorder. There is no evidence to support cannabis to treat opioid use disorder, even in the setting of co-occurring pain.

\section{COMMERCIALIZATION OF CANNABIS FOR MEDICAL USE}

The cannabis industry markets cannabis as safe and natural, though the potency of tetrahydrocannabinol (THC), the psychoactive cannabinoid in cannabis, has increased from approximately $4 \%$ in 1995 to $12 \%$ in $2014 .{ }^{21}$ How potency changes have contributed to harms associated with cannabis use remains unclear. Much of the research examining medical benefits of cannabis studied inhaled cannabis flower with various concentrations of THC/CBD, or synthetic, THC-like cannabinoids, dronabinol and nabilone. Cannabis sold in dispensaries contains varying concentrations of THC/CBD, a result of the cannabinoid extraction process. Available cannabis products include tinctures, lotions, oils, edibles, infused beverages, and inhaled cannabis (smoked flower, vaped, dabbed). Highly concentrated doses of THC, so-called dabs, budder, wax, shatter, etc., are made by extracting pure THC and other cannabinoids from the cannabis plant using solvents like butane or propane. The resultant product is a highly concentrated THC wax which is vaporized for inhalation. Daily use of high-potency cannabis is associated with a nearly fivefold increase in the risk of developing psychotic disorders. ${ }^{22}$ Qualifying conditions to obtain a medical cannabis card vary across states. Some qualifying conditions are supported by research, i.e., the use of plant-derived cannabinoids for pain or childhood-onset seizures. Other qualifying conditions, including HIV/AIDS, hepatitis $\mathrm{C}$ virus infection, migraines, post-traumatic stress disorder, fibromyalgia, are not supported by scientific evidence demonstrating a medical benefit for use. The cannabis industry has capitalized on state-level approval of qualifying medical conditions for cannabis use. In turn, patients increasingly use high-potency cannabis not studied for safety or efficacy. When counseling patients, physicians may provide observational data on 
associated harms with high-potency cannabis use, ${ }^{6}$ but to date, these products have not been studied for medical use. Thus, clear counseling on benefits versus harms cannot be provided to patients seeking information.

\section{DISPENSARIES EMPLOYEES AS PROXY CLINICIANS}

Cannabis is federally classified as a schedule 1 substance with no accepted medical use and high potential for abuse. Due to federal scheduling of cannabis, clinicians do not prescribe cannabis for medical use. Instead, clinicians conduct assessments to certify patients have qualifying medical conditions. Ideally, patients should receive counseling about risks, benefits, and safe administration of medications from their clinicians, in verbal and written form. Due to discrepancies between federal and state cannabis laws, clinicians certifying patients for medical cannabis are not required to make dosing or safety recommendations. Thus, cannabis dispensary workers have become proxy clinicians, recommending products for medical use. In some states, cannabis dispensaries must employ a licensed pharmacist; in other states, dispensary workers lack formal education or certification. In a study of 400 Colorado cannabis dispensaries, $69 \%$ of dispensaries contacted recommended cannabis for pregnancy-related nausea despite evidence supporting an association between maternal cannabis use and neonatal morbidity or death. ${ }^{23,24}$ Rigorously conducted research is urgently needed to study high-potency cannabis for medical use before clinicians can adequately counsel patients. Until then, patients rely on nonmedical dispensary workers, the media, or peers for anecdotal advice, which may be biased by financial gain.

\section{LACK OF STANDARDIZED ASSESSMENTS FOR CONCERNING CANNABIS USE}

Acute or subacute harms associated with cannabis use include increased risk of fatal auto accidents, ${ }^{4}$ acute psychiatric symptoms, such as acute anxiety or psychosis, ${ }^{25}$ and cannabinoid hyperemesis syndrome. ${ }^{25}$ Longer term harms include the development of neurocognitive deficits ${ }^{5}$ and increased risk of addiction. ${ }^{6}$ Assessing patients for cannabis use may reduce future harms. Validated assessment tools are available to clinicians to identify problematic cannabis use and cannabis use disorder, including the Cannabis Use Disorders Identification Test-Revised (CUDIT-R). ${ }^{26}$ Evidenced-based standardized or safe dosages or concentrations, and recommendations for cannabis use are not available for clinicians. When considering current research findings, clinicians should ask about their patient's cannabis use, their indication for use (medical use versus recreational use), and the route of use (inhalation versus edible versus dabbing versus vaping). This information should be documented in the patient's medical record. Follow-up visits should include a query of perceived benefits of cannabis use (i.e., persistent pain reduction) versus immediate or long- term harms associated with use, including the presence of Diagnostic and Statistical Manual of Mental Disorders, 5th Edition (DSM-5) criteria for cannabis use disorder. Including family members in the conversation, when appropriate, may reveal important information regarding the patient's cannabis use. The use of nonjudgmental and non-stigmatizing language is essential to elicit a meaningful history. A longitudinal assessment of patient's cannabis use will identify concerning changes in use necessitating referral to an addiction specialist.

\section{CONCLUSION}

Legalization and commercialization of cannabis have outpaced rigorous scientific research demonstrating the risks and benefits of cannabis use, including the use of highly potent THC products. To reduce public harms, at least three strategies must be employed. First, cannabis tax revenue should be directed at rigorous state-level regulation of cannabis products, including required testing and reporting of $\mathrm{THC} / \mathrm{CBD}$ concentrations, and product marketing which accurately represents the medical benefits of cannabis. Next, the Drug Enforcement Agency (DEA) has issued a single license for the cultivation of cannabis for research in partnership with the National Institute for Drug Abuse (NIDA) and the University of Mississippi. The DEA, FDA, and NIH should continue to strategize ways to allow for rigorous study of high-potency cannabis available for purchase in dispensaries across the USA, including rescheduling of cannabis to facilitate research. Third, largescale efforts should be undertaken to measure cannabis consumption in medical records, similar to measures of alcohol and tobacco consumption, so prospective, longitudinal studies can be conducted to correlate consumption measures with medical and psychiatric outcomes. Until these measures are broadly implemented, physicians should begin by inquiring about cannabis use among their patients, including the route and frequency of cannabis use, and the indication for use. This information should be documented in the medical record and should be discussed during primary care visits, similar to alcohol or tobacco screening. While evidence-based guidelines do not exist for counseling on cannabis use, physicians can use the DSM-5 criteria to diagnose a cannabis use disorder to refer their patient to a higher level of treatment as needed.

Acknowledgments: Dr. Calcaterra would like to acknowledge Drs. Toby and Mort Mower for their generous support of her research efforts.

Corresponding Author: Susan L. Calcaterra, MD, MPH, MS; Department of Medicine, Division of General Internal Medicine University of Colorado School of Medicine, Anschutz Medical Campus, Aurora, CO, USA (e-mail: Susan.calcaterra@cuanschutz.edu).

Funding Information Dr. Cunningham is supported by federal grants R01DA032110 and K24DA036955. Dr. Hopfer is supported by federal grants DA032555, DA035804, and DAO42755. 


\section{Compliance with Ethical Standards:}

Conflict of Interest: Dr. Calcaterra, Dr. Cunningham, and Dr. Hopfer report no conflict of interest.

\section{REFERENCES}

1. Force UPST. Screening and Behavioral Counseling Interventions to Reduce Unhealthy Alcohol Use in Adolescents and Adults: US Preventive Services Task Force Recommendation StatementUSPSTF Recommendation: Screening and Interventions for Unhealthy Alcohol UseUSPSTF Recommendation: Screening and Interventions for Unhealthy Alcohol Use. Jama. 2018;320(18):1899-1909.

2. Counseling and interventions to prevent tobacco use and tobacco-caused disease in adults and pregnant women: U.S. Preventive Services Task Force reaffirmation recommendation statement. Annals of internal medicine. 2009;150(8):551-555.

3. Han B, Compton WM, Blanco C, Jones CM. Trends in and correlates of medical marijuana use among adults in the United States. Drug Alcohol Depend 2018; 186:120-129.

4. Aaronson NK, Ahmedzai S, Bergman B, et al. The European Organization for Research and Treatment of Cancer QLQ-C30: a quality-of-life instrument for use in international clinical trials in oncology. JNCI 1993;85(5):365-376.

5. Pope HG, Jr., Gruber AJ, Hudson JI, Huestis MA, Yurgelun-Todd D Neuropsychological performance in long-term cannabis users. Arch Gen Psychiatry 2001;58(10):909-915.

6. Volkow ND, Baler RD, Compton WM, Weiss SRB. Adverse health effects of marijuana use. N Engl J Med 2014;370(23):2219-2227.

7. Cavazos-Rehg PA, Krauss MJ, Cahn E, et al. Marijuana Promotion Online: an Investigation of Dispensary Practices. Prev Sci 2019;20(2):280-290.

8. Devinsky O, Cross JH, Laux L, et al. Trial of Cannabidiol for DrugResistant Seizures in the Dravet Syndrome. $N$ Engl $\mathrm{J} \mathrm{Med}$ 2017;376(21):2011-2020.

9. Devinsky O, Marsh E, Friedman D, et al. Cannabidiol in patients with treatment-resistant epilepsy: an open-label interventional trial. Lancet Neurol 2016;15(3):270-278.

10. National Academies of Sciences E, Medicine. The health effects of cannabis and cannabinoids: The current state of evidence and recommendations for research. National Academies Press; 2017.

11. Whiting PF, Wolff RF, Deshpande $\mathbf{S}$, et al. Cannabinoids for medical use: a systematic review and meta-analysis. Jama. 2015;313(24):24562473.

12. Vickers AJ, Cronin AM, Maschino AC, et al. Acupuncture for Chronic Pain: Individual Patient Data Meta-analysis. JAMA Intern Med 2012;172(19): 1444-1453.
13. Bradford AC, Bradford WD, Abraham A, Adams GB. Association between US state medical cannabis laws and opioid prescribing in the Medicare Part D population. JAMA Intern Med 2018;178(5):667-672.

14. Wen H, Hockenberry JM. Association of medical and adult-use marijuana laws with opioid prescribing for Medicaid enrollees. JAMA Intern Med 2018;178(5):673-679.

15. Bachhuber MA, Saloner B, Cunningham CO, Barry CL. Medical cannabis laws and opioid analgesic overdose mortality in the United States, 1999-2010. JAMA Intern Med 2014;174(10):1668-1673.

16. Powell D, Pacula RL, Jacobson M. Do medical marijuana laws reduce addictions and deaths related to pain killers? J Health Econ 2018;58:2942.

17. Shover CL, Davis CS, Gordon SC, Humphreys K. Association between medical cannabis laws and opioid overdose mortality has reversed over time. Proc Natl Acad Sci U S A 2019;116(26):12624-12626.

18. Bachhuber MA, Arnsten JH, Cunningham CO, Sohler N. Does Medical Cannabis Use Increase or Decrease the Use of Opioid Analgesics and Other Prescription Drugs? J Addict Med 2018;12(4):259-261.

19. Livne O, Shmulewitz D, Lev-Ran S, Hasin DS. DSM-5 cannabis withdrawal syndrome: Demographic and clinical correlates in U.S. adults. Drug Alcohol Depend 2019;195:170-177.

20. Compton WM, Han B, Jones CM, Blanco C. Cannabis use disorders among adults in the United States during a time of increasing use of cannabis. Drug Alcohol Depend 2019;204:107468.

21. ElSohly MA, Mehmedic Z, Foster S, Gon C, Chandra S, Church JC. Changes in Cannabis Potency Over the Last 2 Decades (1995-2014): Analysis of Current Data in the United States. Biol Psychiatry 2016;79(7):613-619.

22. Di Forti M, Quattrone D, Freeman TP, et al. The contribution of cannabis use to variation in the incidence of psychotic disorder across Europe (EU-GEI): a multicentre case-control study. Lancet Psychiatry 2019;6(5):427-436.

23. Dickson B, Mansfield C, Guiahi M, et al. Recommendations From Cannabis Dispensaries About First-Trimester Cannabis Use. Obstet Gynecol 2018;131(6):1031-1038.

24. Metz TD, Allshouse AA, Hogue CJ, et al. Maternal marijuana use, adverse pregnancy outcomes, and neonatal morbidity. Am $J$ Obstet Gynecol. 2017;217(4):478.e471-478.e478.

25. Monte AA, Shelton SK, Mills E, et al. Acute Illness Associated With Cannabis Use, by Route of Exposure: An Observational Study. Ann Intern Med 2019;170(8):531-537.

26. Adamson SJ, Kay-Lambkin FJ, Baker AL, et al. An improved brief measure of cannabis misuse: the Cannabis Use Disorders Identification Test-Revised (CUDIT-R). Drug Alcohol Depend 2010;110(1-2):137-143.

Publisher's Note Springer Nature remains neutral with regard to jurisdictional claims in published maps and institutional affiliations. 